\title{
Barriers to Innovation in the field of Food Nanotechnology Applications within the European Union
}

\author{
Ralf Greiner \\ Department of Food Technology and Bioprocess Engineering, Max Rubner-Institut
}

Karlsruhe, Germany

Nanotechnology is deemed to be one of the key technologies of the 21st century. It is an area of emerging interest and opens new possibilities for the food industry, including uses in food products, processing and packaging. The market for nanotechnology-derived products for the food sector is predicted to grow rapidly in the coming years. Research activities on applications of nanotechnology in the food sector already include development of improved taste, color, flavor, texture and consistency of food products, increased absorption and bioavailability of nutrients and bioactive compounds, improved quality, shelf-life and safety of food products due to new food packaging materials with improved mechanical, barrier and antimicrobial properties, and nano-sensors for traceability and monitoring the condition of food during transport and storage. Beside technical and safety issues, regulatory and analytical challenges as well as public perception have been identified as barriers to innovation in the field of food nanotechnology applications within the European Union. Nanotechnology has already provoked public concern and debate and is hailed by scientists and corporations for their potential and criticized by environmental and consumer groups because of their risks. Public concern about food nanotechnology applications include a lack of transparency and choice about exposure, risks to health and environment, unfair distribution of risks and benefits and a lack of socially useful applications. It is significant that public concerns extend beyond narrowly defined issues of scientific risk to broader questions over the control, purpose and predictability of nanotechnology's application. 